\title{
Social Media Interventions to Promote HIV Testing, Linkage, Adherence, and Retention: Systematic Review and Meta-Analysis
}

Bolin Cao ${ }^{1,2,3}$, PhD; Somya Gupta ${ }^{4}$, MA; Jiangtao Wang ${ }^{5}$, BA; Lisa B Hightow-Weidman ${ }^{6}$, MD; Kathryn E Muessig ${ }^{7}$, PhD; Weiming Tang ${ }^{2,3,6}$, PhD; Stephen Pan ${ }^{2,3}$, PhD; Razia Pendse ${ }^{4}$, MD; Joseph D Tucker ${ }^{2,3,6}$, MD, PhD

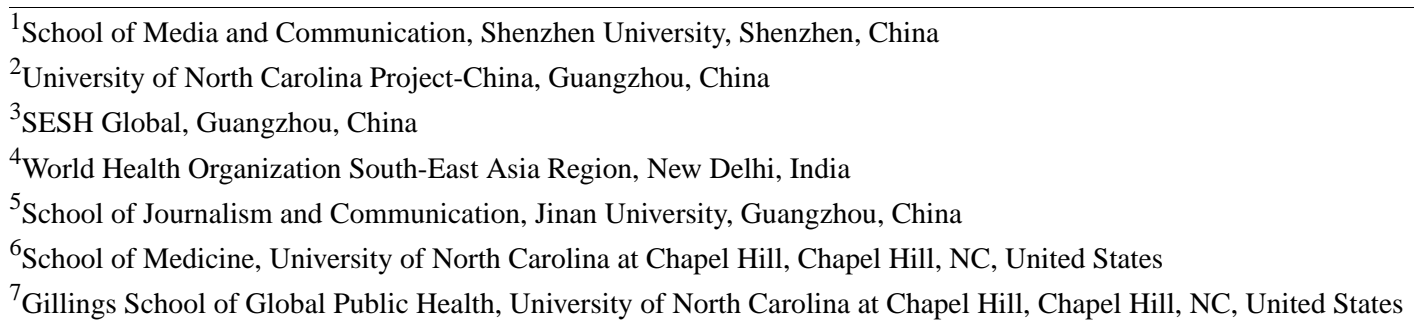

Corresponding Author:

Bolin Cao, $\mathrm{PhD}$

School of Media and Communication

Shenzhen University

No. 3688, Nanhai Avenue, Nanshan District

Shenzhen, 518000

China

Phone: 8613247393943

Fax: 86075526535374

Email: caobolin@szu.edu.cn

\section{Abstract}

Background: Social media is increasingly used to deliver HIV interventions for key populations worldwide. However, little is known about the specific uses and effects of social media on human immunodeficiency virus (HIV) interventions.

Objective: This systematic review examines the effectiveness of social media interventions to promote HIV testing, linkage, adherence, and retention among key populations.

Methods: We used the Preferred Reporting Items for Systematic Reviews and Meta-Analyses (PRISMA) checklist and Cochrane guidelines for this review and registered it on the International Prospective Register of Systematic Reviews, PROSPERO. We systematically searched six databases and three conference websites using search terms related to HIV, social media, and key populations. We included studies where (1) the intervention was created or implemented on social media platforms, (2) study population included men who have sex with men (MSM), transgender individuals, people who inject drugs (PWID), and/or sex workers, and (3) outcomes included promoting HIV testing, linkage, adherence, and/or retention. Meta-analyses were conducted by Review Manager, version 5.3. Pooled relative risk (RR) and 95\% confidence intervals were calculated by random-effects models.

Results: Among 981 manuscripts identified, 26 studies met the inclusion criteria. We found 18 studies from high-income countries, 8 in middle-income countries, and 0 in low-income countries. Eight were randomized controlled trials, and 18 were observational studies. All studies $(\mathrm{n}=26)$ included MSM; five studies also included transgender individuals. The focus of 21 studies was HIV testing, four on HIV testing and linkage to care, and one on antiretroviral therapy adherence. Social media interventions were used to do the following: build online interactive communities to encourage HIV testing/adherence (10 studies), provide HIV testing services (9 studies), disseminate HIV information (9 studies), and develop intervention materials (1 study). Of the studies providing HIV self-testing, $16 \%$ of participants requested HIV testing kits from social media platforms. Existing social media platforms such as Facebook $(n=15)$ and the gay dating app Grindr $(n=10)$ were used most frequently. Data from four studies show that HIV testing uptake increased after social media interventions ( $\mathrm{n}=1283$, RR 1.50, 95\% CI 1.28-1.76). In the studies where social media interventions were participatory, HIV testing uptake was higher in the intervention arm than the comparison arm (n=1023, RR 1.64, 95\% CI 1.19-2.26). 
Conclusions: Social media interventions are effective in promoting HIV testing among MSM in many settings. Social media interventions to improve HIV services beyond HIV testing in low- and middle-income countries and among other key populations need to be considered.

Trial Registration: International Prospective Register of Systematic Reviews (PROSPERO): CRD42016048073; http://www.crd.york.ac.uk/PROSPERO/display_record.php?ID=CRD42016048073 (Archived by WebCite at http://www. webcitation.org/6usLCJK3v)

(J Med Internet Res 2017;19(11):e394) doi: 10.2196/jmir.7997

\section{KEYWORDS}

social media; HIV; MSM; intervention; testing; adherence

\section{Introduction}

Since 2000, global efforts at human immunodeficiency virus (HIV) control have had significant effects. Acquired immune deficiency syndrome (AIDS)-related deaths have been reduced [1], and 19.5 million people are accessing antiretroviral therapy (ART) [2]. To achieve the end of the AIDS epidemic by 2030, $90 \%$ of all people living with HIV (PLWH) should know their HIV status, $90 \%$ of all people with diagnosed HIV infections should receive ART, and $90 \%$ of all people receiving ART should have viral suppression by 2020 [3]. However, in 2016, only $70 \%$ of PLWH globally had been diagnosed, $53 \%$ were receiving ART, and 44\% had achieved viral suppression [4]. Improved efforts are needed to reach PLWH with a comprehensive package of HIV interventions, including HIV testing, linkage to care, ART, and retention. More programs are needed for key populations who are disproportionately affected by HIV and who have difficulty accessing services across the HIV care continuum [5]. The World Health Organization defines key populations as men who have sex with men (MSM), people who inject drugs (PWID), prisoners, sex workers, and transgender people [5,6]. Barriers to HIV interventions among key populations are complex and include persistent stigma and discrimination, punitive laws, and low risk perception [7-9]. Innovative approaches to reach these populations with equitable, accessible, and acceptable services will be essential to achieve 90-90-90 targets [3].

As social media has expanded globally, these platforms have been adopted to deliver HIV interventions, especially for key populations $[10,11]$. Social media is defined as an Internet-based platform that allows the creation and exchange of user-generated content, usually using either mobile or Web-based technologies [12]. Popular social media platforms, such as Facebook and YouTube, have over 1.5 billion monthly active users as of June 2017 [13]. Social media possesses the characteristics of interactivity, allows users to generate content [14], and attracts high user engagement [15]. Specifically, social media can enable convenient access, at any time and place, to information and services on stigmatized diseases such as HIV. In addition, social media can be used to form online communities to seek social support, which is known to improve treatment adherence and uptake of HIV services [16].

Systematic reviews have been conducted recently on the relationship between social media and HIV outcomes $[10,11,17,18]$. These studies generally define social media broadly (eg, to include eHealth and mHealth interventions) or examine usage of social media for a variety of purposes, including recruitment, surveillance, communication, and HIV prevention and treatment. These studies have not focused on social media interventions among key populations [5,6]. Key populations are of special importance because they influence epidemic dynamics and likely have a disproportionate influence on the effectiveness of the response to HIV [5]. To address these gaps in a rapidly growing field, this systematic review and meta-analysis looks at the effectiveness of social media interventions in promoting HIV testing, linkage to care, adherence to treatment, and retention along the HIV care continuum among key populations.

\section{Methods}

\section{Conduct of Systematic Review}

This systematic review was conducted following the Preferred Reporting Items for Systematic Reviews and Meta-Analyses (PRISMA) guidelines [19]. In August 2016, the following databases were systematically searched without restriction on publication date: PubMed, Cochrane Library, Cumulative Index to Nursing and Allied Health Literature (CINAHL), Embase, Scopus, and Sociological Abstracts. Three conference databases (Conference on Retroviruses and Opportunistic Infections; International AIDS Society Conference on HIV Science, and Youth+Tech+Health Conference) were also searched for abstracts for 2015 and 2016.

\section{Search Strategy and Selection Criteria}

The search strategy was designed with a librarian to identify studies regarding social media interventions to promote HIV testing, linkage, adherence, and retention among key populations (Multimedia Appendix 1). It was developed based on key terms, medical subject headings (MeSH) terms, synonyms, and subject headings related to three groups: (1) HIV, (2) social media or social media category or social media platform, including the most popular social networks (websites and apps) and top gay dating apps worldwide selected on the basis of their popularity and number of active users [20-22], and (3) key populations, including MSM, PWID, sex workers, and transgender persons.

Following the PRISMA guidelines [19], key study characteristics such as population, intervention, and outcomes defined the eligibility criteria. In particular, studies were included when (1) study population included key populations such as MSM, PWID, sex workers, and transgender individuals, (2) the intervention was created or implemented on at least one 
social media platform (existing platform or new platform with social networking components), and (3) outcomes included promoting HIV testing, linkage to care and treatment, adherence to ART, or retention. The inclusion criterion did not have any restrictions on geography or setting, except the language of publication as English. Studies without a comparator arm were also included. Studies in which social media were used for marketing/advertising, surveillance, recruitment, or data collection purposes only were excluded. Commentary, protocol, featured article, published articles without full text or sufficient details on interventions or outcomes, and modeling studies were also excluded.

Two investigators independently reviewed all abstracts identified through searches and screened them for eligibility. The full texts of the abstracts that met the eligibility criteria were then reviewed to confirm inclusion in the analysis. Disagreements were resolved through discussion with a third reviewer.

\section{Data Extraction}

Data extraction for the included studies was completed using a standardized extraction form in Microsoft Excel that included the following information: first author, study design (randomized controlled trial [RCT] or observational study), study date, study location, sample size, target population (MSM, PWID, transgender individuals, and/or sex worker), intervention dates/duration, social media platforms, the role of social media, the reach of social media intervention, whether there were interactions in-person or offline events, step in the HIV cascade, and study outcomes. The "role of social media" was categorized into (1) social media used to develop intervention materials for promoting HIV services, (2) social media used to establish virtual peer-mentored or online communities that promote HIV interventions, (3) social media as a platform to offer HIV-related services, such as HIV self-testing kits order and request, and (4) social media as a platform to disseminate HIV-related information [23]. Among various social media interventions, those who used interactive characteristics, tailored contents, or peer influence of social media were considered as participatory social media interventions. For studies with a comparator arm, data were abstracted on the type of interventions, participants, and outcomes for both the intervention and the comparator arm.

\section{Quality Assessment}

Two reviewers assessed the quality of the included studies using the checklist tool in Sanderson et al [24], and a third reviewer collated the results. For each study, the following six domains were used to assess risk of bias: (1) methods for selecting study participants, (2) methods for measuring exposure and outcome variables, (3) design-specific source of bias, (4) method of control confounding, (5) statistical methods, and (6) other biases (including conflict of interest and disclosure of funding sources). The Cochrane Collaboration's recommendations [25] were used to categorize each of the six domains as "low risk of bias" ("+") or "high risk of bias" (“-”).

\section{Statistical Analysis}

For RCTs meeting the inclusion criteria, pooled relative risks (RR) were used to compare the participants in the intervention and the comparator arm with respect to HIV testing rates among total participants. Pooled RR were also used to compare HIV testing outcomes at baseline and post social media interventions. Data from studies on HIV self-sampling were pooled to summarize (1) the proportion of total participants requesting HIV self-sampling services, (2) the proportion of participants requesting HIV self-sampling who returned their test kits, and (3) HIV positivity rates. Meta-analyses were conducted by Review Manager, version 5.3. Pooled RR and 95\% confidence intervals (CI) were calculated using random-effects models.

\section{Results}

\section{Study Characteristics}

Of the 981 articles and abstracts, 26 studies met our inclusion criteria [26-51] (Figure 1). Of the 26 studies, eight were RCTs $[28,33,41,42,46,47,49,50]$ and 18 were observational studies [26,27,29-32,34-40,43-45,48,51] (Table 1). These studies were implemented in 10 countries from 2007-2015 and published from 2011-2016. In total, 18 studies were from four high-income countries as defined by the World Bank $[28,29,31-38,40,42-45,47,48,50], 10$ studies were from the United States [28,33-35,38,42,43,45,47,50], five from the United Kingdom [29,31,32,36,48], two each from Australia [40,44], China [46,51], and Thailand [26,27], and one each from Taiwan [37], India [41], Peru [49], Mexico [30], and Guatemala [39]. The reach of social media interventions varied from 55 to over 17,000 individuals.

\section{Key Populations}

MSM were the primary population included in the reviewed social media interventions. All of the included studies contained MSM populations, and five also covered transgender individuals $[26,27,35,42,46]$. In three studies, MSM with the following specific characteristics were included: young MSM [28], young black MSM [47], and MSM living with HIV [33].

\section{Social Media Platforms}

Existing social and sexual networking sites and gay-specific websites/apps were used for interventions. Fifteen studies used Facebook [26-29,32,33,35-37,39,41,44,47,49,50] and 10 studies used Grindr, a social networking app catering to MSM [29,32,34-36,38-40,45,48]. Other generic social networking platforms included YouTube, Twitter, and QQ, and other social networking sites or apps catering to MSM included Gaydar, Jack'd, Scruff, A4A, and Radar. Six studies used both social and sexual networking sites as intervention platforms $[29,32,35,36,39,51]$. Five studies created their own social media platform to provide HIV services [26-28,30,33].

\section{Role of Social Media}

In one study, social media was used as a crowdsourcing tool to develop intervention materials (video) for promotion of HIV testing [46]. The study showed that the crowdsourced video $\operatorname{arm}(114 / 307,37.1 \%)$ had similar results as the social marketing $\operatorname{arm}(111 / 317,35.0 \%)$ in promoting HIV tests, but the cost of crowdsourced intervention was less than the social marketing intervention per first-time HIV test (US \$131 vs US \$238 per person) and per new HIV diagnosis (US\$415 vs US $\$ 799$ per person). 
Figure 1. PRISMA chart showing study selection process.

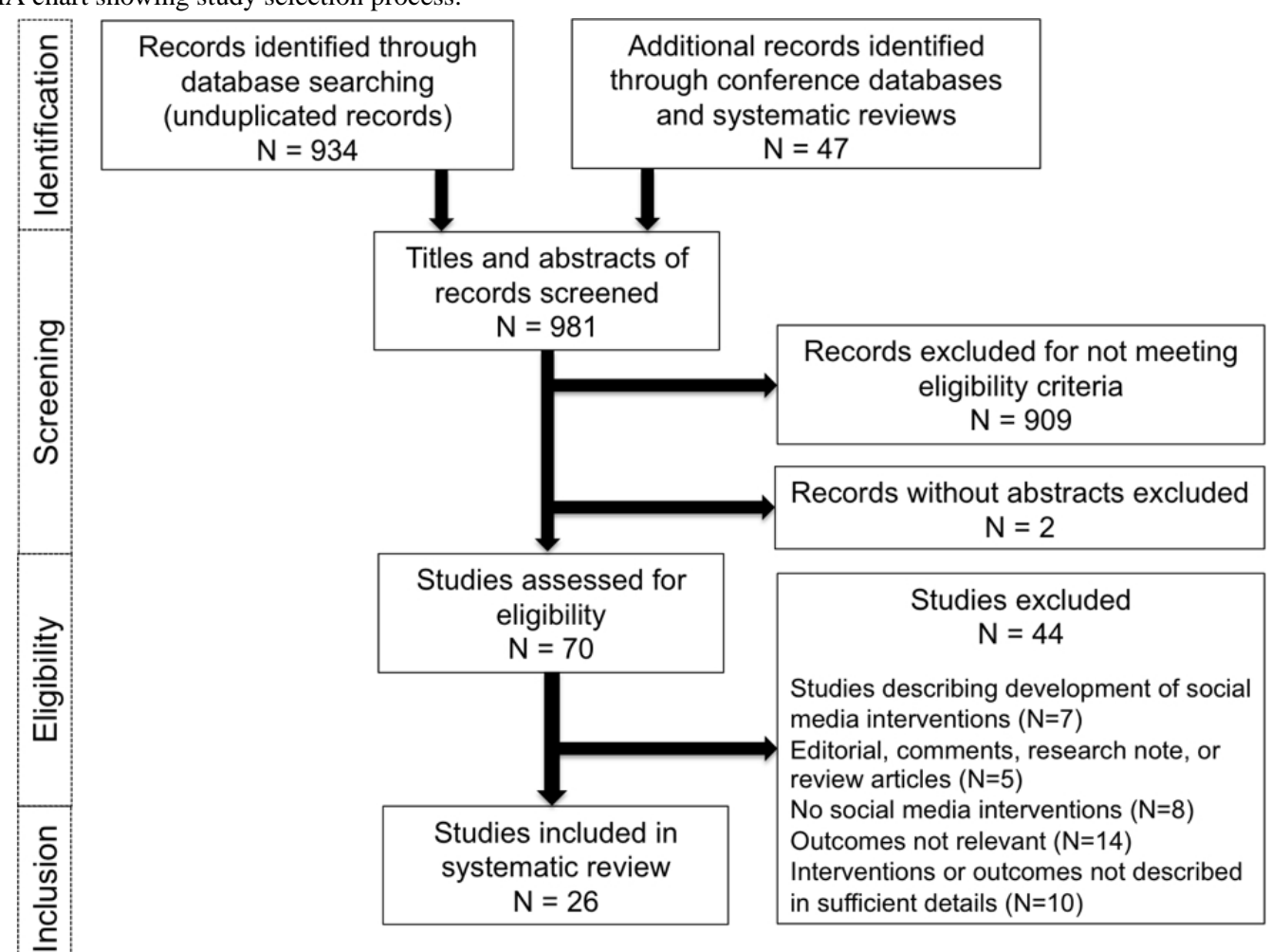

Ten studies reported using social media to establish virtual communities where HIV services (testing and adherence) were promoted $[26,33,37,40,42,43,45,47,49,50]$. While two studies built their own platforms to form interactive communities $[26,33]$, the remaining studies established these communities on Facebook, popular gay dating apps or existing chat rooms $[37,40,42,43,45,47,49,50]$. Interventions provided in the communities included online HIV counseling, educating people about HIV and importance of HIV testing, referral for HIV testing, and answering questions related to HIV. HIV testing uptake was higher in virtual communities that emphasized peer-to-peer interaction among participants $[37,49]$.

Social media also served as a platform for delivering services such as HIV self-testing kits, HIV self-sampling, or home-based HIV testing (9 studies) [27,29,31,32,34,36,44,48,50]. MSM were often recruited through a broadcast message as a pop-up or a personal message or promotional banner on social media, and the most popular platforms were Facebook and Grindr
[29,32,34]. Finally, HIV-related information was disseminated using social media in nine studies [28-30,35,38,39,41,44,51]. Some studies tailored the content to be distributed on social media by user's race/ethnicity and age based on quantitative data and evaluated through community advisory boards and youth advisory boards [28]. Other studies trained outreach health educators on using gay-specific apps to provide MSM with HIV education, counseling, and testing information [38,39].

In three studies, social media played more than one role $[29,44,50]$. In 14 studies, the online activities that were a part of the social media interventions were augmented with offline events or in-person interaction [26,27,29-32,35,36,38,39,44,48,49,51]. For example, during or post social media interventions, HIV testing were provided at clinics or home [27,36,38,51] and in studies on HIV test home-sampling, test results were conveyed to participants over the phone or via messages [29]. 
Table 1. Characteristics of the studies included in the systematic review $(n=26)$.

\begin{tabular}{|c|c|c|c|c|c|c|c|c|c|}
\hline Study & Study design & $\begin{array}{l}\text { Study } \\
\text { location }\end{array}$ & $\begin{array}{l}\text { Sample } \\
\text { size }\end{array}$ & $\begin{array}{l}\text { Target } \\
\text { population }\end{array}$ & $\begin{array}{l}\text { Intervention } \\
\text { period/ } \\
\text { duration }\end{array}$ & $\begin{array}{l}\text { Social media } \\
\text { platforms }\end{array}$ & $\begin{array}{l}\text { Role of } \\
\text { social } \\
\text { media }\end{array}$ & $\begin{array}{l}\text { Interaction } \\
\text { in-person } \\
\text { or offline }\end{array}$ & $\begin{array}{l}\text { Step in the } \\
\text { HIV cascade }\end{array}$ \\
\hline $\begin{array}{l}\text { Anand } 2015 \\
{[26]}\end{array}$ & Observational & Thailand & 11,120 & $\begin{array}{l}\text { MSM \& } \\
\text { transgender } \\
\text { individuals }\end{array}$ & $\begin{array}{l}2011.09- \\
2014.12 \text { (on- } \\
\text { going) }\end{array}$ & $\begin{array}{l}\text { Facebook, Line, } \\
\text { "Adam's Love" } \\
\text { online platform }\end{array}$ & $\begin{array}{l}\text { Build com- } \\
\text { munity }^{\mathrm{a}}\end{array}$ & Yes & $\begin{array}{l}\text { HIV testing } \\
\text { and linkage } \\
\text { to care }\end{array}$ \\
\hline $\begin{array}{l}\text { Anand } 2016 \\
{[27]}\end{array}$ & Observational & Thailand & 97 & $\begin{array}{l}\text { MSM \& } \\
\text { transgender } \\
\text { individuals }\end{array}$ & $\begin{array}{l}2015.12- \\
2016.05\end{array}$ & $\begin{array}{l}\text { Facebook, Line, } \\
\text { "Adam's Love" } \\
\text { online platform }\end{array}$ & Deliver kits $^{b}$ & Yes & HIV testing \\
\hline $\begin{array}{l}\text { Bauermeister } \\
2015 \text { [28] }\end{array}$ & RCT & US & 130 & Young MSM & 30 days & $\begin{array}{l}\text { Facebook; "Get } \\
\text { connected" plat- } \\
\text { form }\end{array}$ & Disseminate $^{c}$ & No & HIV testing \\
\hline $\begin{array}{l}\text { Brady } 2014 \\
\text { [29] }\end{array}$ & Observational & UK & 17,629 & MSM & $\begin{array}{l}\text { Phase 1: } \\
\text { 2013.01- } \\
\text { 2013.09; } \\
\text { Phase 2: } \\
\text { 2013.11- } \\
2014.03\end{array}$ & $\begin{array}{l}\text { Grindr, Gaydar, } \\
\text { Manhunt, Face- } \\
\text { book, Twitter }\end{array}$ & $\begin{array}{l}\text { Deliver kits } \\
\text { (blood) \& } \\
\text { disseminate }\end{array}$ & Yes & $\begin{array}{l}\text { HIV testing } \\
\text { and linkage } \\
\text { to care }\end{array}$ \\
\hline $\begin{array}{l}\text { Buzdugan } \\
2016[30]\end{array}$ & Observational & Mexico & 61 & MSM & 5 weeks & $\begin{array}{l}\text { Online game } \\
\text { app }\end{array}$ & Disseminate & Yes & HIV testing \\
\hline $\begin{array}{l}\text { Elliot } 2012 \\
{[31]}\end{array}$ & Observational & UK & 321 & MSM & $\begin{array}{l}2011.11 .07- \\
2012.01 .11\end{array}$ & Gaydar & Deliver kits & Yes & HIV testing \\
\hline $\begin{array}{l}\text { Elliot } 2016 \\
{[32]}\end{array}$ & Observational & UK & 17,361 & MSM & 2012-2014 & $\begin{array}{l}\text { Gaydar, Grindr, } \\
\text { Facebook }\end{array}$ & $\begin{array}{l}\text { Deliver kits } \\
\text { (oral) }\end{array}$ & Yes & $\begin{array}{l}\text { HIV testing } \\
\text { and linkage } \\
\text { to care }\end{array}$ \\
\hline $\begin{array}{l}\text { Horvath } 2013 \\
\text { [33] }\end{array}$ & $\mathrm{RCT}$ & US & 123 & $\begin{array}{l}\text { MSM living } \\
\text { with HIV }\end{array}$ & $\begin{array}{l}2011.02- \\
2011.04\end{array}$ & $\begin{array}{l}\text { Facebook; } \\
\text { "Thrive with } \\
\text { me" platform }\end{array}$ & $\begin{array}{l}\text { Build com- } \\
\text { munity }\end{array}$ & No & $\begin{array}{l}\text { ART adher- } \\
\text { ence }\end{array}$ \\
\hline $\begin{array}{l}\text { Huang } 2015 \\
{[34]}\end{array}$ & Observational & US & $\begin{array}{l}16,328 \\
(112 \text { in } \\
\text { survey) }\end{array}$ & MSM & $\begin{array}{l}\text { Phase 1: } \\
\text { 2014.04.17- } \\
\text { 2014.05.29; } \\
\text { Phase 2: } \\
\text { 2014.10.13- } \\
2014.11 .11\end{array}$ & Grindr & $\begin{array}{l}\text { Deliver kits } \\
\text { (oral) }\end{array}$ & No & HIV testing \\
\hline $\begin{array}{l}\text { Hyden } 2016 \\
\text { [35] }\end{array}$ & $\begin{array}{l}\text { Observational } \\
\text { study }\end{array}$ & US & 274 & $\begin{array}{l}\text { MSM \& } \\
\text { transgender } \\
\text { individuals }\end{array}$ & $\begin{array}{l}2013.03- \\
2017.12 \text { (on- } \\
\text { going) }\end{array}$ & $\begin{array}{l}\text { Facebook, } \\
\text { Scruff, Grindr, } \\
\text { Online game } \\
\text { App }\end{array}$ & Disseminate & Yes & HIV testing \\
\hline $\begin{array}{l}\text { Jones } 2015 \\
{[36]}\end{array}$ & Observational & UK & 305 & MSM & $\begin{array}{l}2014.11 .29- \\
2014.11 .30 \\
(2 \text { days })\end{array}$ & $\begin{array}{l}\text { Facebook, } \\
\text { Grindr, Squirt }\end{array}$ & $\begin{array}{l}\text { Deliver kits } \\
\text { (blood) }\end{array}$ & Yes & HIV testing \\
\hline Ko 2013 [37] & Observational & Taiwan & 1037 & MSM & $\begin{array}{l}2011.04- \\
2011.09\end{array}$ & Facebook & $\begin{array}{l}\text { Build com- } \\
\text { munity }\end{array}$ & No & HIV testing \\
\hline $\begin{array}{l}\text { Lampkin } 2016 \\
\text { [38] }\end{array}$ & Observational & US & 903 & MSM & $\begin{array}{l}2012.10- \\
2013.03 \\
2013.10- \\
2014.03\end{array}$ & Grindr & Disseminate & Yes & HIV testing \\
\hline $\begin{array}{l}\text { Mendizabal- } \\
\text { Burastero } \\
2016 \text { [39] }\end{array}$ & Observational & Guatemala & 7244 & MSM & $\begin{array}{l}2014.07- \\
2015.12\end{array}$ & $\begin{array}{l}\text { Facebook, } \\
\text { Twitter, Grindr, } \\
\text { WhatsApp }\end{array}$ & Disseminate & Yes & $\begin{array}{l}\text { HIV testing } \\
\text { and linkage } \\
\text { to care }\end{array}$ \\
\hline $\begin{array}{l}\text { Munro } 2016 \\
{[40]}\end{array}$ & Observational & Australia & & MSM & $\begin{array}{l}\text { Not men- } \\
\text { tioned }\end{array}$ & Grindr & $\begin{array}{l}\text { Build com- } \\
\text { munity }\end{array}$ & No & HIV testing \\
\hline $\begin{array}{l}\text { Patel } 2016 \\
{[41]}\end{array}$ & $\mathrm{RCT}$ & India & 244 & MSM & $\begin{array}{l}2015.02- \\
2015.05\end{array}$ & $\begin{array}{l}\text { Facebook, } \\
\text { WhatsApp }\end{array}$ & Disseminate & No & HIV testing \\
\hline
\end{tabular}




\begin{tabular}{|c|c|c|c|c|c|c|c|c|c|}
\hline Study & Study design & $\begin{array}{l}\text { Study } \\
\text { location }\end{array}$ & $\begin{array}{l}\text { Sample } \\
\text { size }\end{array}$ & $\begin{array}{l}\text { Target } \\
\text { population }\end{array}$ & $\begin{array}{l}\text { Intervention } \\
\text { period/ } \\
\text { duration }\end{array}$ & $\begin{array}{l}\text { Social media } \\
\text { platforms }\end{array}$ & $\begin{array}{l}\text { Role of } \\
\text { social } \\
\text { media }\end{array}$ & $\begin{array}{l}\text { Interaction } \\
\text { in-person } \\
\text { or offline }\end{array}$ & $\begin{array}{l}\text { Step in the } \\
\text { HIV cascade }\end{array}$ \\
\hline $\begin{array}{l}\text { Rhodes } 2016 \\
\text { [42] }\end{array}$ & RCT & US & 1292 & $\begin{array}{l}\text { MSM \& } \\
\text { transgender } \\
\text { individuals }\end{array}$ & $\begin{array}{l}2013.07- \\
2014.06\end{array}$ & $\begin{array}{l}\text { Adam4Adam, } \\
\text { BlackGayChat, } \\
\text { Craigslist, } \\
\text { Gay.com }\end{array}$ & $\begin{array}{l}\text { Build com- } \\
\text { munity }\end{array}$ & No & HIV testing \\
\hline $\begin{array}{l}\text { Rhodes } 2011 \\
\text { [43] }\end{array}$ & Observational & US & 509 & MSM & $\begin{array}{l}2009.02- \\
2009.07\end{array}$ & $\begin{array}{l}\text { Online chat } \\
\text { room }\end{array}$ & $\begin{array}{l}\text { Build com- } \\
\text { munity }\end{array}$ & No & HIV testing \\
\hline $\begin{array}{l}\text { Roberts } 2015 \\
\text { [44] }\end{array}$ & Observational & Australia & 503 & MSM & $\begin{array}{l}2013.11 \& \\
2014.07\end{array}$ & $\begin{array}{l}\text { Facebook, } \\
\text { YouTube, Twit- } \\
\text { ter }\end{array}$ & $\begin{array}{l}\text { Deliver kits } \\
\& \text { dissemi- } \\
\text { nate }\end{array}$ & Yes & HIV testing \\
\hline Sun 2015 [45] & Observational & US & 2709 & MSM & $\begin{array}{l}2013.08- \\
2014.02\end{array}$ & $\begin{array}{l}\text { A4A Radar, } \\
\text { Grindr, Jack'd, } \\
\text { Scruff }\end{array}$ & $\begin{array}{l}\text { Build com- } \\
\text { munity }\end{array}$ & No & HIV testing \\
\hline $\begin{array}{l}\text { Tang } 2016 \\
{[46]}\end{array}$ & $\mathrm{RCT}$ & China & 721 & $\begin{array}{l}\text { MSM \& } \\
\text { transgender } \\
\text { individuals }\end{array}$ & 2014.09 & $\begin{array}{l}\text { MSM dating } \\
\text { websites }\end{array}$ & $\begin{array}{l}\text { Develop ma- } \\
\text { terials }^{\mathrm{d}}\end{array}$ & No & HIV testing \\
\hline $\begin{array}{l}\text { Washington } \\
2016 \text { [47] }\end{array}$ & $\mathrm{RCT}$ & US & 142 & $\begin{array}{l}\text { Young black } \\
\text { MSM }\end{array}$ & $\begin{array}{l}\text { Not men- } \\
\text { tioned }\end{array}$ & Facebook & $\begin{array}{l}\text { Build com- } \\
\text { munity }\end{array}$ & No & HIV testing \\
\hline $\begin{array}{l}\text { West } 2015 \\
\text { [48] }\end{array}$ & Observational & UK & 55 & MSM & $\begin{array}{l}2014.11(5 \\
\text { days) }\end{array}$ & Grindr & Deliver kits & Yes & HIV testing \\
\hline $\begin{array}{l}\text { Young } 2015 \\
\text { [49] }\end{array}$ & RCT & Peru & 556 & MSM & $\begin{array}{l}\text { Phase 1: } \\
\text { 2012.03.19- } \\
\text { 2012.06.11; } \\
\text { Phase 2: } \\
\text { 2012.09.26- } \\
2012.12 .19\end{array}$ & Facebook & $\begin{array}{l}\text { Build com- } \\
\text { munity }\end{array}$ & Yes & HIV testing \\
\hline $\begin{array}{l}\text { Young } 2013 \\
{[50]}\end{array}$ & $\mathrm{RCT}$ & US & 112 & MSM & $\begin{array}{l}2010.09- \\
2011.01\end{array}$ & Facebook & $\begin{array}{l}\text { Build com- } \\
\text { munity \& de- } \\
\text { liver kits }\end{array}$ & No & HIV testing \\
\hline Zou 2013 [51] & Observational & China & 429 & MSM & $\begin{array}{l}2007.06- \\
2007.08\end{array}$ & $\begin{array}{l}\text { Gay dating \& } \\
\text { volunteer web- } \\
\text { sites, QQ, on- } \\
\text { line gay chat- } \\
\text { room }\end{array}$ & Disseminate & Yes & HIV testing \\
\hline
\end{tabular}

\footnotetext{
a"Build community" is when social media is used to establish virtual peer-mentored or online communities that promote HIV interventions.

b"Deliver kits" is when social media serves as a platform to deliver newly designed or extant evidence-based HIV intervention.

c“Disseminate" is when social media is used as a platform to disseminate HIV-related information.

d“Develop materials" is when social media is used to develop intervention materials for promoting HIV services.
}

\section{Uptake of HIV Testing and HIV Home Sampling}

We found 25 studies that used social media interventions to promote HIV testing [26-32,34-51] among MSM or MSM and transgender individuals, while one study used it for improving ART adherence [33]. Of these 25 studies, seven were RCTs $[27,40,41,45,46,48,49]$ and the remaining 18 were observational studies [26,27,29-32,34-40,43-45,48,51]. Four studies were pooled to compare the rate of HIV testing at baseline and postintervention [37,41-43]. The meta-analysis showed that the HIV testing rate significantly increased after the social media interventions were provided ( $\mathrm{RR}=1.50,95 \%$ CI $1.28-1.76, I^{2}$ $66 \%$; Figure 2).

Data from five RCTs show that when social media interventions were participatory $[28,42,46,49,50]$, HIV testing rates were significantly higher (RR 1.64, 95\% CI 1.19-2.26, $I^{2} 75 \%$; Figure
3) than in the comparative arm, where there were no social media interventions, or the social media interventions provided general health information or were not participatory. Of the seven RCTs, Patel et al [41] was excluded since both arms were interventional arms while Washington et al [47] was excluded from the analysis due to nonavailability of complete data. Similar to included studies, in Washington et al [47], those in the intervention arm (HIV testing video + chat) were seven times more likely to test for HIV than those in control group receiving standard HIV text information (study not included in pooled RR due to incomplete data).

Four studies in the United Kingdom and United States used social media to offer HIV self-testing services to MSM $[29,31,32,50]$ (Tables 2,3, and 4). On average, $15.65 \%$ of the participants requested HIV testing kits $(\mathrm{n}=67,054 ; 95 \% \mathrm{CI}$ 15.37-15.92), ranging from $15.50 \%$ to $36.36 \%$ (data from three studies) $[30,31,49]$. Nearly $57 \%$ of the participants who 
requested test kits returned them $(n=24,703 ; 95 \% \quad \mathrm{CI}$ 55.92-57.16). On average, the HIV positivity rate was $1.51 \%$ among those who received results $(n=13,956 ; 95 \%$ CI 1.32-1.73) (data from three studies [29,31,32,34]).

\section{Quality Assessment}

There was a high risk of bias in methods for selecting study participants in 15 studies [27,29-32,34-36,38-40,42-44,48] and in measuring exposure and outcome bias in 13 studies
[26,29,31,34-36,38-40,44,45,48,51]. Design-specific source of bias was present in 10 studies [27,28,30,36,39,41,45,46,49,50]. Concerns regarding control confounding bias were in 14 studies $[26,27,29-32,34,35,37,40,44,45,48,51]$ and concerns of statistical methods in 15 studies [26,29-32,34-36,38-40,44,48,49,51] (Multimedia Appendix 2). Only two studies had low risk of bias in all the 6 dimensions $[33,47]$. See Multimedia Appendix 3 for results of the included studies.

Figure 2. Comparison between studies with baseline and postintervention data on HIV testing $(n=4)$.

\begin{tabular}{|c|c|c|c|c|c|c|c|c|c|}
\hline Study or Subgroup & \multicolumn{2}{|c|}{ Post-intervention } & \multicolumn{2}{|c|}{ Baseline } & \multicolumn{3}{|c|}{ Risk Ratio } & \multicolumn{2}{|c|}{$\begin{array}{c}\text { Risk Ratio } \\
\text { M-H, Random, 95\% CI }\end{array}$} \\
\hline Ko 2013 & 219 & 499 & 150 & 501 & $27.5 \%$ & $1.47[1.24,1.73]$ & & & $=$ \\
\hline Patel 2016 & 216 & 339 & 122 & 353 & $27.7 \%$ & $1.84[1.56,2.17]$ & & & $=$ \\
\hline Rhodes 2011 & 187 & 315 & 154 & 346 & $29.3 \%$ & $1.33[1.15,1.55]$ & & & $=$ \\
\hline Rhodes 2016 & 57 & 130 & 42 & 130 & $15.5 \%$ & $1.36[0.99,1.86]$ & & & $=-$ \\
\hline Total $(95 \% \mathrm{Cl})$ & & 1283 & & 1330 & $100.0 \%$ & $1.50[1.28,1.76]$ & & & $\boldsymbol{1}$ \\
\hline Total events & 679 & & 468 & & & & & & \\
\hline $\begin{array}{l}\text { Heterogeneity. Tau }{ }^{2} \\
\text { Test for overall effect }\end{array}$ & $\begin{array}{l}0.02 ; \mathrm{Chi}^{2}= \\
\mathrm{Z}=4.99(\mathrm{P}\end{array}$ & $\begin{array}{l}89, \mathrm{df} \\
0.0000\end{array}$ & $=3(P=$ & $=0.03 \mathrm{~J}$ & $1^{2}=66 \%$ & & 0.01 & ${ }^{1} .1$ Baseline $^{1}$ & $1_{\text {Post-intervention }}^{10} 100$ \\
\hline
\end{tabular}

Figure 3. Comparison of HIV testing uptake between the intervention arm and the comparison arm in the RCTs ( $\mathrm{n}=5$ ).

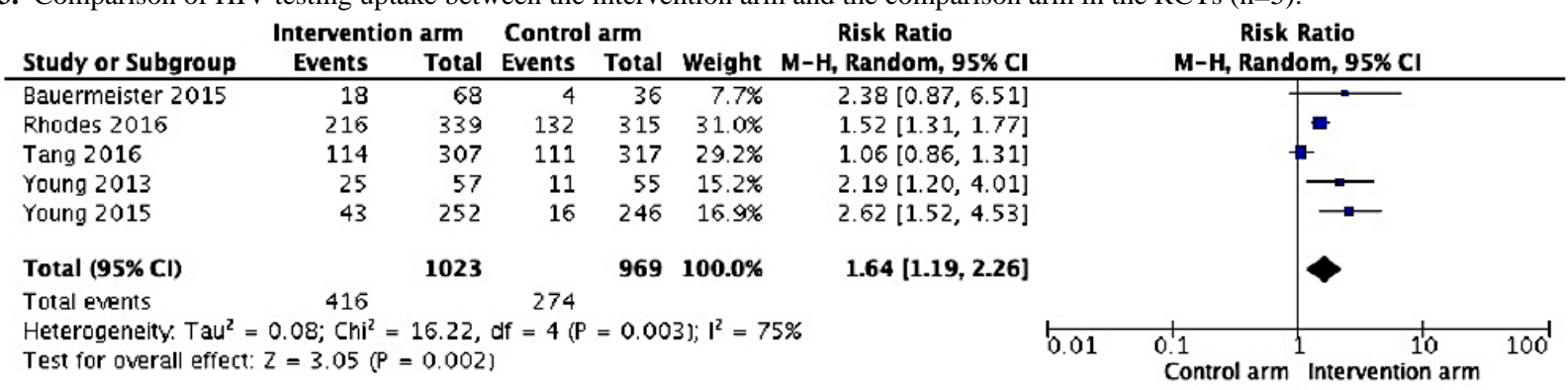

Table 2. Studies that use social media to provide HIV self-testing services $(n=3)$ : Request for HIV test kits.

\begin{tabular}{llll}
\hline Study & Total participants/webpage views & Request testing kit & Request percentage (95\% CI) \\
\hline Elliot 2012 [31] & 363 & 132 & $36.36(31.58-41.43)$ \\
Elliot 2016 [32] & 66,579 & 10,323 & 36 \\
Young 2013 [50] & 112 & $15.50(15.23-15.78)$ & $32.14(24.21-41.26)$ \\
Total & 67,054 & 15.691 & $(15.37-15.92)$ \\
\hline
\end{tabular}

Table 3. Studies that use social media to provide HIV self-testing services $(n=4)$ : Return rate of test kits.

\begin{tabular}{llll}
\hline Study & Request for testing kit & Returned testing kit & Return percentage (95\% CI) \\
\hline Brady 2014 [29] & 14,212 & 8187 & 73 \\
Elliot 2012 [31] & 132 & $57.61(56.79-58.42)$ & $55.30(46.79-63.52)$ \\
Elliot 2016 [32] & 10,323 & 11 & $55.18(54.22-56.13)$ \\
Young 2013 [50] & 36 & 13,967 & $30.56(18.00-46.86)$ \\
Total & 24,703 & $56.54(55.92-57.16)$ & \\
\hline
\end{tabular}


Table 4. Studies that use social media to provide HIV self-testing services $(n=4)$ : HIV positivity rate.

\begin{tabular}{llll}
\hline Study & Total participants tested & HIV positive & Positive rate (95\% CI) \\
\hline Brady 2014 [29] & 8187 & 111 & $1.36(1.13-1.63)$ \\
Elliot 2012 [31] & 73 & 4 & $5.48(2.15-13.26)$ \\
Elliot 2016 [32] & 5696 & 96 & $1.69(1.38-2.05)$ \\
Total & 13,956 & 211 & $1.51(1.32-1.73)$ \\
\hline
\end{tabular}

\section{Discussion}

\section{Principal Findings}

Social media have played varied roles and have been used in multiple settings to promote public health interventions. For example, social media was found to be effective in promoting physical activity [52] and smoking cessation [53]. Previous reviews have also found that social media interventions can improve HIV-related outcomes such as promoting HIV testing and linkage to care $[10,11,17,18]$. This systematic review shows that social media interventions are being used to promote HIV testing among MSM and transgender populations. In the studies included, HIV testing uptake among this key population group increased after implementation of social media interventions. Also, HIV testing uptake was particularly higher when the social media interventions were participatory and peer-driven. These findings are consistent with other studies [54,55] and support implementation of social media interventions that reach MSM populations and promote MSM participation.

HIV testing is the main focus of social media interventions along the HIV care continuum and is critical for achieving the first Joint United Nations Programme on HIV/AIDS (UNAIDS) 90-90-90 target [3]. Four studies found that HIV self-testing kits distributed through social media led to large numbers of requests for and return of test kits. World Health Organization guidelines strongly recommend HIV self-testing as an additional approach to HIV testing services [56]. One study on HIV self-testing included in this review concludes that providing self-testing through social media is acceptable and cost-effective [34]. Cost per person tested was US \$39 and US \$26 of this cost was attributed to test kits [57]. This is comparable to other research that found self-testing (US \$9.23) costs lower than facility-based testing (US \$11.84) in Malawi [58]. The current studies that use social media to reach hard-to-reach populations and offer HIV self-sampling or self-testing services were implemented in high-income countries where social media penetration rates are high. More usability research on HIV self-testing using social media is needed in low- and middle-income countries. Meanwhile, all included articles reported using social media to promote HIV testing, except that one study aimed to enhance ART adherence [33]. In addition to promoting HIV testing, future research should explore how social media can be leveraged to promote other HIV care services.

Social media interventions that were participatory and peer-led resulted in higher HIV testing rates compared with those that had no social media interventions or social media interventions that did not include interactive characteristics. Engaging people to participate in the interventions can increase their perceived relevance and importance of the activities [59]. Participatory strategies of social media interventions, such as tailoring intervention contents to user's characteristics [28,49] and encouraging peer interactions [49,50] and community contributions [46], can increase the likelihood for participants to link to HIV care and services. Provided that participatory and peer-led social media interventions allow the organizers to receive feedback from the users and can motivate users to take actions, future social media interventions should adopt established strategies or develop new strategies to improve engagement and interaction with participants.

Among the studies included in this review, a variety of social media platforms were used for promoting HIV services. They included existing online social and sexual networking platforms and standalone HIV-focused websites or apps. Over half of the studies used Facebook or one of the globally or nationally popular gay dating apps. Existing platforms may be useful for HIV interventions as they often have wide reach, high user engagement and retention, and attract specific key populations that would otherwise be difficult to recruit [60]. On the other hand, five of the 26 studies developed standalone platforms. Such platforms could be used to design more tailored and innovative interventions, but using only standalone platforms may have the drawback of poor retention and limited coverage [61]. Few studies used other popular social media platforms such as Twitter and YouTube, partly because such platforms are often used as knowledge, news, or video sharing platforms but not to develop long-term relationships for continuous service provision [62]. Research is needed to assess the appropriateness and effectiveness of these platforms to deliver HIV-specific interventions for key populations.

All studies in this review primarily focused on MSM populations. This may be due to the early adoption and popularity of social and sexual networking sites among this population. Online sex seeking sites and apps for MSM provide an important channel for seeking social support and meeting new sexual partners $[63,64]$. While use of these platforms has been associated with increased risk behaviors for HIV [65,66], they have also been effectively used for promotion of HIV testing $[47,67]$. Although several studies included transgender people in addition to MSM, results were not disaggregated and studies were not powered to evaluate differences in transgender populations. The feasibility and acceptability of social media interventions and the appropriate social media platforms to deliver HIV services within networks of PWID and sex workers is also underresearched. Given disparities along the prevention and care continuum with these populations [68] and strong within-group networks [69], the potential of using social media in these populations should be actively explored. 


\section{Limitations}

This systematic review found that studies on social media intervention have several limitations. First, the current research's methodological scoring was low due to the fact that the majority of the studies were exploratory and descriptive. Of the 26 studies, 24 had high or unclear risk of bias for at least one of the bias items in the methodological quality assessment. The high risk of bias indicates relatively low quality of studies on social media interventions, hindering more pooled analysis and causing possible bias of this meta-analysis. Second, there was also a variety of control groups used in the seven RCTs on HIV testing. For example, in the control groups, either no interventions were provided or social media was used to provide general health-related information or promote HIV services without interactive features and peer-leaders. Third, some studies used apps to improve linkage and retention in care, but for one-way monitoring of patients instead of bidirectional interactions.

This systematic review also has its own limitations. First, it was restricted to English language publications and was not able to cover studies in other languages. However, an empirical analysis found that this was not associated with bias [70]. Second, due to the heterogeneity of intervention types and measures in outcomes, we had difficulties pooling certain data. Some pooled results were largely driven by a few studies. This meta-analysis was not able to compare effects of social media intervention on MSM versus transgender individuals and through standalone platform versus existing platforms. Last, currently many studies on this topic are underway, so the state-of-the-science will continue to grow rapidly over the next few years.

\section{Future Studies}

This systematic review shows the effectiveness of using social media interventions to improve HIV testing among MSM population and has implications for both future research and public policy. Gaps should be filled on using social media to promote HIV services beyond HIV testing and among key populations beyond MSM. Moreover, how to maximize the use of social media to promote HIV service needs further exploration. In addition, given that social media interventions have been found to be effective, there is an opportunity for national programs to leverage social media to support scale-up of such interventions. In particular, low- and middle-income countries, where mobile usage is rising, seem to have relatively few social media interventions.

\section{Conclusion}

A total of 26 articles were identified in this systematic review to examine the role of rapidly expanding social media in improving access to HIV-related interventions and its effect in promoting HIV services among key populations. Social media can contribute to creating innovative intervention programs, disseminating intervention information, building virtual communities, and especially promoting HIV self-testing and self-sampling. Social media interventions were effective in increasing HIV testing rates, especially in high-income countries, aimed at MSM. To achieve the goal of 90-90-90 by 2020 and ultimately end AIDS by 2030, adapting these social media interventions in low- to middle-income countries and other key populations may be useful.

\section{Acknowledgments}

Support of this work was provided by the National Institutes of Health (National Institute of Allergy and Infectious Diseases 1R01AI1 14310); UNC-South China STD Research Training Centre (Fogarty International Centre 1D43TW009532); UNC Center for AIDS Research (National Institute of Allergy and Infectious Diseases 5P30AI050410); National Social Science Foundation of China (16CXW031); and the Bill \& Melinda Gates Foundation to the MeSH Consortium (BMGF-OPP1120138). This publication was also supported by Grant Number UL1TR001111 from the National Center for Advancing Translational Sciences (NCATS) at the National Institutes of Health.

We acknowledge Jennifer S Walker, UNC, a Chapel Hill librarian, for conducting the initial search for studies, and Gabriella Stein, undergraduate student, UNC, Chapel Hill, for her support at the early stage of this study.

The findings and conclusions in this study are those of the authors and do not necessarily represent the views of the World Health Organization.

\section{Conflicts of Interest}

None declared.

\section{Multimedia Appendix 1}

Search terms.

[PDF File (Adobe PDF File), 24KB-Multimedia Appendix 1]

\section{Multimedia Appendix 2}

Quality assessment of the included studies. 


\section{Multimedia Appendix 3}

Results of included studies.

[PDF File (Adobe PDF File), 34KB-Multimedia Appendix 3]

\section{References}

1. Joint UN Programme on HIV/AIDS (UNAIDS). Prevention Gap Report. Geneva, Switzerland URL: http://www.unaids.org/ sites/default/files/media asset/2016-prevention-gap-report en.pdf [accessed 2017-11-05] [WebCite Cache ID 6ukNT13cH]

2. UNAIDS. UNAIDS announces 18.2 million people on antiretroviral therapy, but warns that 15-24 years of age is a highly dangerous time for young women. URL: http://www.unaids.org/en/resources/presscentre/pressreleaseandstatementarchive/ 2016/november/20161121 PR get-on-the-fast-track [accessed 2017-09-16] [WebCite Cache ID 6tX2aDSGD]

3. Joint UN Programme on HIV/AIDS (UNAIDS). 90-90-90 - An ambitious treatment target to help end the AIDS epidemic. 2017 Jan 01. URL: http://www.unaids.org/en/resources/documents/2017/90-90-90 [accessed 2017-08-18] [WebCite Cache ID 6snZis7u6]

4. UNAIDS. Ending AIDS: Progress towards the 90-90-90 targets. 2017. URL: http://www.unaids.org/sites/default/files/ media asset/Global AIDS update 2017 en.pdf [accessed 2017-09-18] [WebCite Cache ID 6tZLC1E1o]

5. World Health Organization. Consolidated guidelines on HIV prevention, diagnosis, treatment and care for key populations. 2014. URL: http://apps.who.int/iris/bitstream/10665/128048/1/9789241507431_eng.pdf [accessed 2017-09-18] [WebCite Cache ID 6tZLPyEtE]

6. World Health Organization. WHO Definition of key terms. 2013 Jun. URL: http://www.who.int/hiv/pub/guidelines/arv2013/ intro/keyterms/en/ [accessed 2017-08-11] [WebCite Cache ID 6tZLMqK3o]

7. Altman D, Aggleton P, Williams M, Kong T, Reddy V, Harrad D, et al. Men who have sex with men: stigma and discrimination. Lancet 2012 Jul 28;380(9839):439-445. [doi: 10.1016/S0140-6736(12)60920-9] [Medline: 22819652]

8. Decker MR, Crago A, Chu SKH, Sherman SG, Seshu MS, Buthelezi K, et al. Human rights violations against sex workers: burden and effect on HIV. Lancet 2015 Jan 10;385(9963):186-199 [FREE Full text] [doi: 10.1016/S0140-6736(14)60800-X] [Medline: 25059943]

9. Kamarulzaman A, Altice FL. Challenges in managing HIV in people who use drugs. Curr Opin Infect Dis 2015 Feb;28(1):10-16 [FREE Full text] [doi: 10.1097/QCO.0000000000000125] [Medline: 25490106]

10. Garett R, Smith J, Young SD. A Review of Social Media Technologies Across the Global HIV Care Continuum. Curr Opin Psychol 2016 Jun 01;9:56-66 [FREE Full text] [doi: 10.1016/j.copsyc.2015.10.024] [Medline: 26925455]

11. Taggart T, Grewe ME, Conserve DF, Gliwa C, Roman IM. Social Media and HIV: A Systematic Review of Uses of Social Media in HIV Communication. J Med Internet Res 2015 Nov 02;17(11):e248 [FREE Full text] [doi: 10.2196/jmir.4387] [Medline: 26525289]

12. Margetts H, John P, Hale S, Yasseri T. How social media shape collective action. New Jersey, US: Princeton University Press; 2015.

13. Constine J. TechCrunch. Facebook now has 2 billion monthly users... and responsibility URL: https://techcrunch.com/2017/ 06/27/facebook-2-billion-users/ [accessed 2017-08-18] [WebCite Cache ID 6snaB5jFi]

14. Obar JA, Wildman SS. Social media definition and the governance challenge: An introduction to the special issue. Telecommunications Policy 2015;39(9):745-750. [doi: 10.1016/j.telpol.2015.07.014]

15. Saberi P, Johnson M. Correlation of Internet Use for Health Care Engagement Purposes and HIV Clinical Outcomes Among HIV-Positive Individuals Using Online Social Media. J Health Commun 2015;20(9):1026-1032 [FREE Full text] [doi: 10.1080/10810730.2015.1018617] [Medline: 26120890]

16. Mo P, Coulson N. Exploring the communication of social support within virtual communities: a content analysis of messages posted to an online HIV/AIDS support group. Cyberpsychol Behav 2008 Jun;11(3):371-374. [doi: 10.1089/cpb.2007.0118] [Medline: 18537512]

17. Ghosh D, Krishnan A, Gibson B, Brown S, Latkin CA, Altice FL. Social Network Strategies to Address HIV Prevention and Treatment Continuum of Care Among At-risk and HIV-infected Substance Users: A Systematic Scoping Review. AIDS Behav 2017 Apr;21(4):1183-1207. [doi: 10.1007/s10461-016-1413-y] [Medline: 27125244]

18. Muessig K, Nekkanti M, Bauermeister J, Bull S, Hightow-Weidman L. A systematic review of recent smartphone, Internet and Web 2.0 interventions to address the HIV continuum of care. Curr HIV/AIDS Rep 2015 Mar;12(1):173-190 [FREE Full text] [doi: 10.1007/s11904-014-0239-3] [Medline: 25626718]

19. Moher D, Liberati A, Tetzlaff J, Altman D, PRISMA Group. Preferred reporting items for systematic reviews and meta-analyses: the PRISMA statement. Int J Surg 2010;8(5):336-341 [FREE Full text] [doi: 10.1016/j.ijsu.2010.02.007] [Medline: 20171303]

20. Statista. 2017. Global active usage penetration of leading social networks as of February 2017 URL: https://www.statista.com/ statistics/274773/global-penetration-of-selected-social-media-sites/ [accessed 2017-09-09] [WebCite Cache ID 6tZMIiuiq]

21. Digital Trends. 2017. Find love or have a fling with the best LGBT dating apps for iPhone and Android URL: https://www. digitaltrends.com/mobile/best-lgbt-dating-apps-iphone-android/ [accessed 2017-09-19] [WebCite Cache ID 6tZMQm4go] 
22. Johnson N. Plugin Group. 2016. The top 15 most popular social media sites in 2016 URL: https://plugingroup.com/ top-15-popular-social-media-sites-november-2016/ [accessed 2017-09-19] [WebCite Cache ID 6tZMVB2xp]

23. Tso L, Tang W, Li H, Yan H, Tucker J. Social media interventions to prevent HIV: A review of interventions and methodological considerations. Curr Opin Psychol 2016 Jun 01;9:6-10 [FREE Full text] [doi: 10.1016/j.copsyc.2015.09.019] [Medline: 26516632]

24. Sanderson S, Tatt ID, Higgins JPT. Tools for assessing quality and susceptibility to bias in observational studies in epidemiology: a systematic review and annotated bibliography. Int J Epidemiol 2007 Jun;36(3):666-676. [doi: 10.1093/ije/dym018] [Medline: 17470488]

25. The Cochrane Collaboration. 2011 Mar. Cochrane Handbook for Systematic Reviews of Interventions (Version 5.1.0) URL: http://handbook-5-1.cochrane.org/ [accessed 2017-11-06] [WebCite Cache ID 6ukR01O78]

26. Anand T, Nitpolprasert C, Ananworanich J, Pakam C, Nonenoy S, Jantarapakde J, et al. Innovative strategies using communications technologies to engage gay men and other men who have sex with men into early HIV testing and treatment in Thailand. J Virus Erad 2015 Apr 01;1(2):111-115 [FREE Full text] [Medline: 27482400]

27. Anand T, Nitpolprasert C, Kerr S, Apornpong T, Ananworanich J, Phanuphak P, et al. Factors influencing and associated with the decision to join in Thailand's first online supervised HIV self-testing and counselling initiative. J Int AIDS Soc 2016;19((8Suppl 7)):21487. [doi: 10.7448/IAS.19.8.21487]

28. Bauermeister JA, Pingel ES, Jadwin-Cakmak L, Harper GW, Horvath K, Weiss G, et al. Acceptability and preliminary efficacy of a tailored online HIV/STI testing intervention for young men who have sex with men: the Get Connected! program. AIDS Behav 2015 Oct;19(10):1860-1874 [FREE Full text] [doi: 10.1007/s10461-015-1009-y] [Medline: 25638038]

29. Brady M, Nardone A, Buenaventura E, Qureshi F, Edwardes D, Kelly P. Home HIV sampling linked to national HIV testing campaigns: A novel approach to improve HIV diagnosis. HIV Med 2014;15:7-8.

30. Buzdugan R. Evaluation of a pilot game-based intervention to prevent HIV among young men who have sex with men in Mexico City. 2016 Presented at: Youth + Tech + Heal Conference; 2016; San Francisco, CA.

31. Elliot E, McCowan A, McCormak S, Rossi M, Van Every T. O20 Home sampling through social network websites: can we reduce undiagnosed HIV? Sex Transm Infect 2012 May 23;88(Suppl 1):A8. [doi: 10.1136/sextrans-2012-050601a.20]

32. Elliot E, Rossi M, McCormack S, McOwan A. Identifying undiagnosed HIV in men who have sex with men (MSM) by offering HIV home sampling via online gay social media: a service evaluation. Sex Transm Infect 2016 Sep;92(6):470-473. [doi: 10.1136/sextrans-2015-052090] [Medline: 27221475]

33. Horvath K, Oakes JM, Rosser BRS, Danilenko G, Vezina H, Amico KR, et al. Feasibility, acceptability and preliminary efficacy of an online peer-to-peer social support ART adherence intervention. AIDS Behav 2013 Jul;17(6):2031-2044 [FREE Full text] [doi: 10.1007/s10461-013-0469-1] [Medline: 23553347]

34. Huang E, Marlin RW, Young SD, Medline A, Klausner JD. Using Grindr, a Smartphone Social-Networking Application, to Increase HIV Self-Testing Among Black and Latino Men Who Have Sex With Men in Los Angeles, 2014. AIDS Educ Prev 2016 Aug;28(4):341-350. [doi: 10.1521/aeap.2016.28.4.341] [Medline: 27427928]

35. Hyden C. "Heads or Tails?" Online interactive graphic novel for HIV/STI prevention: Content development and opportunities for adaptation. 2015 Presented at: Youth+Health+Tech Conference; 2015; San Francisco, CA.

36. Jones M, Aderogba K, Drunis L. Providing rapid HIV testing in their homes for men who have sex with men, recruited via social media. HIV Medicine 2015;16:50-51.

37. Ko N, Hsieh C, Wang M, Lee C, Chen C, Chung A, et al. Effects of Internet popular opinion leaders (iPOL) among Internet-using men who have sex with men. J Med Internet Res 2013 Feb 25;15(2):e40 [FREE Full text] [doi:

10.2196/jmir.2264] [Medline: 23439583]

38. Lampkin D, Crawley A, Lopez TP, Mejia CM, Yuen W, Levy V. Reaching Suburban Men Who Have Sex With Men for STD and HIV Services Through Online Social Networking Outreach: A Public Health Approach. J Acquir Immune Defic Syndr 2016 May 01;72(1):73-78. [doi: 10.1097/QAI.0000000000000930] [Medline: 27097365]

39. Mendizabal-Burastero R. Reaching the unreachable: MSM recruitment strategy using social networks to HIV prevention services in Guatemala city. 2016 Presented at: International AIDS Society AIDS Conference; 2016; Durban, South Africa.

40. Munro T, Rabiem L, Gray J. Accessing gay men: transitioning from physical venues to online spaces. 2016 Presented at: International AIDS Society AIDS Conference; 2016; Durban, South Africa.

41. Patel V. CHALO! A social media based peer-delivered intervention increases HIV testing in men who have sex with men in Mumbai, India: a randomized trial. 2016 Presented at: International AIDS Society AIDS Conference; 2016; Durban, South Africa.

42. Rhodes SD, McCoy TP, Tanner AE, Stowers J, Bachmann LH, Nguyen AL, et al. Using Social Media to Increase HIV Testing Among Gay and Bisexual Men, Other Men Who Have Sex With Men, and Transgender Persons: Outcomes From a Randomized Community Trial. Clin Infect Dis 2016 Dec 01;62(11):1450-1453 [FREE Full text] [doi: 10.1093/cid/ciw127] [Medline: 26980878]

43. Rhodes S, Vissman A, Stowers J, Miller C, McCoy T, Hergenrather K, et al. A CBPR partnership increases HIV testing among men who have sex with men (MSM): outcome findings from a pilot test of the CyBER/testing internet intervention. Health Educ Behav 2011 Jun;38(3):311-320 [FREE Full text] [doi: 10.1177/1090198110379572] [Medline: 21393625] 
44. Roberts N, Holden J, Duck T, Kitchener S. Health promotion 'on steroids': the value of an experiential approach to promote rapid HIV testing in NSW, Australia. Public Health Res Pract 2015 Mar 30;25(2):e2521522 [FREE Full text] [doi: 10.17061/phrp2521522] [Medline: 25848740]

45. Sun CJ, Stowers J, Miller C, Bachmann LH, Rhodes SD. Acceptability and feasibility of using established geosocial and sexual networking mobile applications to promote HIV and STD testing among men who have sex with men. AIDS Behav 2015 Mar;19(3):543-552 [FREE Full text] [doi: 10.1007/s10461-014-0942-5] [Medline: 25381563]

46. Tang W, Han L, Best J, Zhang Y, Mollan K, Kim J, et al. Crowdsourcing HIV Test Promotion Videos: A Noninferiority Randomized Controlled Trial in China. Clin Infect Dis 2016 Dec 01;62(11):1436-1442 [FREE Full text] [doi: 10.1093/cid/ciw171] [Medline: 27129465]

47. Washington T, Applewhite S. Using social media as a platform to direct young Black men who have sex with men to an intervention to increase HIV testing. 2016 Presented at: International AIDS Society AIDS Conference; 2016; Durban, South Africa.

48. West R, Okecha E, Forbes K. Keeping “app” to date: using geolocation apps to signpost to local sexual health services. Sex Transm Infect 2015 Jun 18:A32-A33. [doi: 10.1136/sextrans-2015-052126.96]

49. Young S, Cumberland W, Nianogo R, Menacho L, Galea J, Coates T. The HOPE social media intervention for global HIV prevention in Peru: a cluster randomised controlled trial. Lancet HIV 2015 Jan;2(1):e27-e32 [FREE Full text] [doi: 10.1016/S2352-3018(14)00006-X] [Medline: 26236767]

50. Young SD, Cumberland WG, Lee S, Jaganath D, Szekeres G, Coates T. Social networking technologies as an emerging tool for HIV prevention: a cluster randomized trial. Ann Intern Med 2013 Sep 03;159(5):318-324 [FREE Full text] [doi: 10.7326/0003-4819-159-5-201309030-00005] [Medline: 24026317]

51. Zou H, Wu Z, Yu J, Li M, Ablimit M, Li F, et al. Internet-facilitated, voluntary counseling and testing (VCT) clinic-based HIV testing among men who have sex with men in China. PLoS One 2013;8(2):e51919 [FREE Full text] [doi:

10.1371/journal.pone.0051919] [Medline: 23418417]

52. Cavallo DN, Tate DF, Ries AV, Brown JD, DeVellis RF, Ammerman AS. A social media-based physical activity intervention: a randomized controlled trial. Am J Prev Med 2012 Nov;43(5):527-532 [FREE Full text] [doi: 10.1016/j.amepre.2012.07.019] [Medline: 23079176]

53. Duke J, Hansen H, Kim A, Curry L, Allen J. The use of social media by state tobacco control programs to promote smoking cessation: a cross-sectional study. J Med Internet Res 2014 Jul 10;16(7):e169 [FREE Full text] [doi: 10.2196/jmir.3430] [Medline: 25014311]

54. Moorhead SA, Hazlett DE, Harrison L, Carroll JK, Irwin A, Hoving C. A new dimension of health care: systematic review of the uses, benefits, and limitations of social media for health communication. J Med Internet Res 2013 Apr 23;15(4):e85 [FREE Full text] [doi: 10.2196/jmir.1933] [Medline: 23615206]

55. Noar S, Pierce L, Black H. Can computer-mediated interventions change theoretical mediators of safer sex? A meta-analysis. Hum Commun Res 2010;36(3):261-297. [doi: 10.1111/j.1468-2958.2010.01376.x]

56. World Health Organization. 2016. Guidelines on HIV self-testing and partner notification URL: http://apps.who.int/iris/ bitstream/10665/251655/1/9789241549868-eng.pdf?ua=1 [accessed 2017-09-18] [WebCite Cache ID 6tZNkhl0a]

57. Huang E, Marlin R, Medline A, Young S, Daniels J. Cost-effectiveness of HIV self-testing promotion through Grindr, a smartphone social networking application. Sex Transm Infect 2015;91(Suppl 2):A226.

58. Maheswaran H, Petrou S, MacPherson P, Choko AT, Kumwenda F, Lalloo DG, et al. Cost and quality of life analysis of HIV self-testing and facility-based HIV testing and counselling in Blantyre, Malawi. BMC Med 2016 Feb 19;14:34 [FREE Full text] [doi: 10.1186/s12916-016-0577-7] [Medline: 26891969]

59. Petty RE, Cacioppo JT. Issue involvement can increase or decrease persuasion by enhancing message-relevant cognitive responses. Journal of Personality and Social Psychology 1979;37(10):1915-1926. [doi: 10.1037/0022-3514.37.10.1915]

60. Hightow-Weidman LB, Muessig KE. New media challenges and opportunities. Sex Transm Infect 2017 Aug;93(5):309-310. [doi: 10.1136/sextrans-2016-052673] [Medline: 27888207]

61. Maher CA, Lewis LK, Ferrar K, Marshall S, De BI, Vandelanotte C. Are health behavior change interventions that use online social networks effective? A systematic review. J Med Internet Res 2014 Feb 14;16(2):e40 [FREE Full text] [doi: 10.2196/jmir.2952] [Medline: 24550083]

62. Lee EJ, Oh SY. Seek and You Shall Find? How Need for Orientation Moderates Knowledge Gain from Twitter Use. J Commun 2013 Jun 24;63(4):745-765. [doi: 10.1111/jcom.12041]

63. Grov C, Breslow AS, Newcomb ME, Rosenberger JG, Bauermeister JA. Gay and bisexual men's use of the Internet: research from the 1990s through 2013. J Sex Res 2014;51(4):390-409 [FREE Full text] [doi: 10.1080/00224499.2013.871626] [Medline: 24754360]

64. Mustanski B, Greene GJ, Ryan D, Whitton SW. Feasibility, acceptability, and initial efficacy of an online sexual health promotion program for LGBT youth: the Queer Sex Ed intervention. J Sex Res 2015;52(2):220-230. [doi: 10.1080/00224499.2013.867924] [Medline: 24588408]

65. Benotsch EG, Kalichman S, Cage M. Men who have met sex partners via the Internet: prevalence, predictors, and implications for HIV prevention. Arch Sex Behav 2002 Apr;31(2):177-183. [Medline: 11974643] 
66. McFarlane M, Bull SS, Rietmeijer CA. The Internet as a newly emerging risk environment for sexually transmitted diseases. JAMA 2000 Jul 26;284(4):443-446. [Medline: 10904506]

67. Cao B, Liu C, Durvasula M, Tang W, Pan S, Saffer A, et al. Social Media Engagement and HIV Testing Among Men Who Have Sex With Men in China: A Nationwide Cross-Sectional Survey. J Med Internet Res 2017 Jul 19;19(7):e251 [FREE Full text] [doi: 10.2196/jmir.7251] [Medline: 28724510]

68. Risher K, Mayer KH, Beyrer C. HIV treatment cascade in MSM, people who inject drugs, and sex workers. Curr Opin HIV AIDS 2015 Nov;10(6):420-429 [FREE Full text] [Medline: 26352393]

69. Tucker J, Peng H, Wang K, Chang H, Zhang S, Yang L, et al. Female sex worker social networks and STI/HIV prevention in South China. PLoS One 2011;6(9):e24816 [FREE Full text] [doi: 10.1371/journal.pone.0024816] [Medline: 21931856]

70. Morrison A, Polisena J, Husereau D, Moulton K, Clark M, Fiander M, et al. The effect of English-language restriction on systematic review-based meta-analyses: a systematic review of empirical studies. Int J Technol Assess Health Care 2012 Apr;28(2):138-144. [doi: 10.1017/S0266462312000086] [Medline: 22559755]

\title{
Abbreviations
}

AIDS: acquired immune deficiency syndrome

ART: antiretroviral therapy

CINAHL: Cumulative Index to Nursing and Allied Health Literature

HIV: human immunodeficiency virus

MSM: men who have sex with men

PLWH: people living with HIV

PRISMA: Preferred Reporting Items for Systematic Reviews and Meta-Analyses

PWID: people who inject drugs

RCT: randomized controlled trial

RR: relative risk

UNAIDS: The Joint United Nations Programme on HIV/AIDS

UNC: University of North Carolina

\author{
Edited by G Eysenbach; submitted 08.05.17; peer-reviewed by TED Yeo, C Khosropour, C Mejia, J Andriesen; comments to author \\ 29.07.17; revised version received 19.09.17; accepted 11.10.17; published 24.11.17 \\ Please cite as: \\ Cao B, Gupta S, Wang J, Hightow-Weidman LB, Muessig KE, Tang W, Pan S, Pendse R, Tucker JD \\ Social Media Interventions to Promote HIV Testing, Linkage, Adherence, and Retention: Systematic Review and Meta-Analysis \\ J Med Internet Res 2017;19(11):e394 \\ URL: http://www.jmir.org/2017/11/e394/ \\ doi: 10.2196/jmir.7997 \\ PMID: 29175811
}

(CBolin Cao, Somya Gupta, Jiangtao Wang, Lisa B Hightow-Weidman, Kathryn E Muessig, Weiming Tang, Stephen Pan, Razia Pendse, Joseph D. Tucker. Originally published in the Journal of Medical Internet Research (http://www.jmir.org), 24.11.2017. This is an open-access article distributed under the terms of the Creative Commons Attribution License (https://creativecommons.org/licenses/by/4.0/), which permits unrestricted use, distribution, and reproduction in any medium, provided the original work, first published in the Journal of Medical Internet Research, is properly cited. The complete bibliographic information, a link to the original publication on http://www.jmir.org/, as well as this copyright and license information must be included. 